\title{
Taxonomic Study of Family Doliolidae (Urochordata: Thaliacea: Doliolida) from Korea
}

\author{
Sunwoo Kim, Jeahyun Lee and Chang-Bae Kim* \\ Department of Green Life Science, Sangmyung University, Seoul 110-743, Korea
}

\begin{abstract}
Four species in two genera of the family Doliolidae are identified and re-described in Korean waters: Doliolum denticulatum Quoy and Gaimard, 1834, Doliolum nationalis Borgert, 1894, Dolioletta gegenbauri (Uljanin, 1884), and Dolioletta tritonis (Herdman, 1888). Dolioletta gegenbauri is newly recorded in Korean fauna. A key to species of the family Doliolidae in Korea is provided.
\end{abstract}

Keywords: taxonomy, Urochordata, Doliolidae, Doliolum, Dolioletta, Korea

\section{INTRODUCTION}

The Doliolidae is one of 4 families of the order Doliolida in thaliaceans. The thaliaceans are filter feeders and free-swimming marine holoplanktons. Doliolids have no less than six different and successive morphological stages (Godeaux, 1998b). About twenty species in four genera are currently known in this family worldwide (Godeaux, 1998b).

The taxonomic study on Korean Doliolidae has not been attempted. Three species of the genus Doliolum: Doliolum denticulatum Quay and Gaimard, Doliolum nationalis Borgert and Doliolum tritonis Herdman have only been recorded in the "List of Animals in Korea" (The Korean Society of Systematic Zoology, 1997) without descriptions and remarks. Therefore, systematic study with descriptions and figures is necessary.

\section{MATERIALS AND METHODS}

The specimens were collected using NORPAC net of $300 \mu \mathrm{m}$ mesh aperture. They were fixed and stored in $4 \%$ buffered formalin or $80 \%$ ethyl alcohol. And then they were examined with stereoscopic microscope (Leica MZ16; Carl zeiss with AxioCam HRc). The body length was measured with objective micrometer and ocular micrometer from the oral aperture to atrial aperture. All specimens examined were in the gonozooid or phorozooid stages. They were deposited in the Department of Green Life Science, Sangmyung University.

Abbreviation used in this study is as follows: M, muscle band.

\footnotetext{
*To whom correspondence should be addressed

Tel: 82-2-2287-5288, Fax: 82-2-2287-0070

E-mail: evodevo@smu.ac.kr
}

\section{SYSTEMATIC ACCOUNTS}

Family Doliolidae Bronn, 1862

Genus Doliolum Quoy and Gaimard, 1834

Doliolum denticulatum Quoy and Gaimard, 1834

(Fig. 1A, B)

Doliolum denticulatum Quoy and Gaimard, 1834, p. 559 (cited from Kott, 2005); Madin, 1991, p. 107, fig. T-3; Kott, 2005, p. 204.

Material examined. 30 individuals, South Sea, $34^{\circ} 05^{\prime} \mathrm{N}$ $127^{\circ} 96^{\prime} \mathrm{E}, 2$, 4 Oct. 2007.

Description. Gonozooid stage: 3-6 mm long, transparent barrel-shaped body with eight muscle bands (Fig. 1A). Branchial septum strongly arched from M II dorsally to M III ventrally (Fig. 1A). Endostyle short, from M II to M IV (Fig. 1B). Dextral arched intestine, alimentary canal open at front side of M V, anus present at M VI (Fig. 1B). Neural ganglion at M III 3/4 (Fig. 1A). Testis horizontal and long, start in front of M III, sometimes beyond M II (Fig. 1A).

Distribution. Widespread in all oceans from $40^{\circ} \mathrm{N}$ to $40^{\circ} \mathrm{S}$.

Doliolum nationalis Borgert, 1894 (Fig. 1C-E)

Doliolum nationalis Borgert, 1894, p. 1. (cited from Berrill, 1950); Berrill, 1950, pp. 282-284, fig. 102.

Material examined. 30 individuals, Yellow Sea, $35^{\circ} 58^{\prime} \mathrm{N}$ $125^{\circ} 32^{\prime}$ E, 21 Aug., 20 Nov. 2008; 15 individuals, East Sea, $35^{\circ} 31^{\prime} \mathrm{N} 129^{\circ} 33^{\prime} \mathrm{E}, 21$ May 2009.

Description. Phorozooid stage: About $3 \mathrm{~mm}$ long, transparent barrel-shaped body with eight muscle bands (Fig. 1C). Branchial septum arched from M II dorsally to just in front of M V ventrally (Fig. 1C). Endostyle short, from M II to M IV (Fig. 1D). Dextral arched intestine, alimentary canal open at front side of M V, anus present at M VI(Fig. 1D). Neural ganglion 

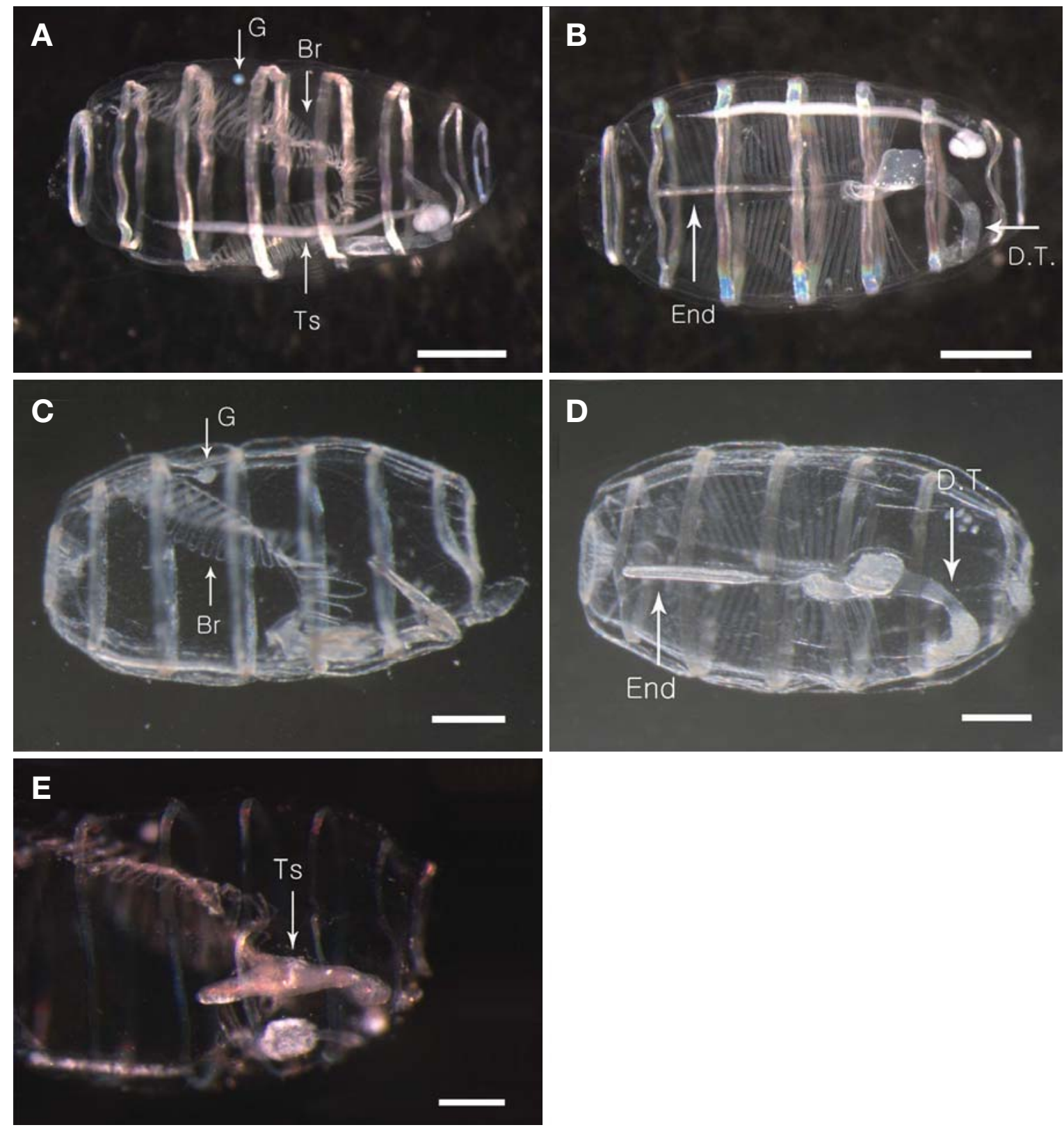

Fig. 1. A-B. Doliolum denticulatum, gonozooid stage. A, lateral view; B, ventral view, C-E. Doliolum nationalis, phorozooid stage. C, lateral view; $D$, ventral view; $E$, testis of gonozooid stage. (Br, branchial septum; D.T., intestine; End, endostyle; $G$, neural ganglion; Ts, testis). Scale bars=1 mm (A, B), $0.5 \mathrm{~mm}(\mathrm{C}-\mathrm{E})$.

at M III 3/4 (Fig. 1C).

Gonozooid stage: Testis horizontal and short, start behind M IV, sometimes just behind M III (Fig. 1E).

Remarks. D. nationalis is very similar to D. denticulatum except branchial septum extending from M II dorsally to M IV $1 / 2$ ventrally.

Distribution. North and Central Atlantic Ocean, Mediterranean
Sea, Red Sea, subtropical southwestern Atlantic Ocean, tropical Indian and West Pacific Ocean.

1*Genus Dolioletta Borgert, 1894

2*Dolioletta gegenbauri (Uljanin, 1884) (Fig. 2A-D)

Doliolum gegenbaueri Uljanin, 1884, p. 1 (cited from Berrill, 1950).

\footnotetext{
$1 *$ 바다술통사촌속 (신칭), ${ }^{2}$ *바다술통사촌 (신칭)
} 


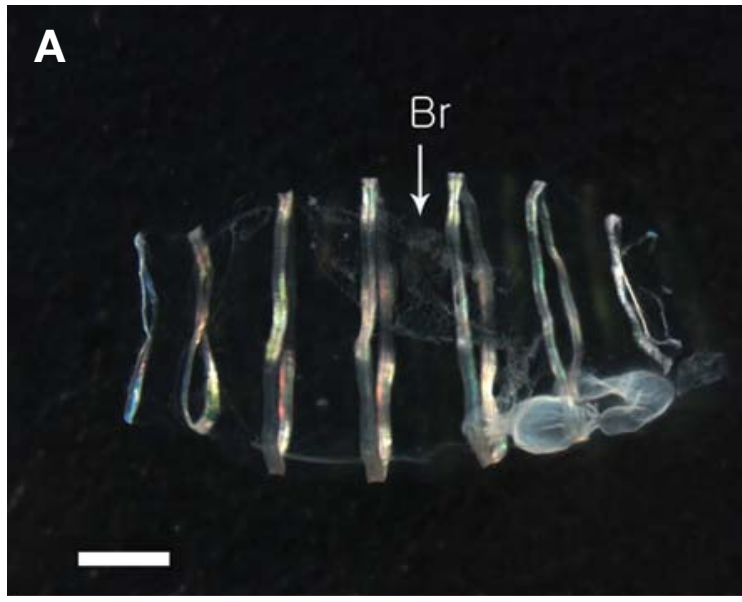

\section{B}
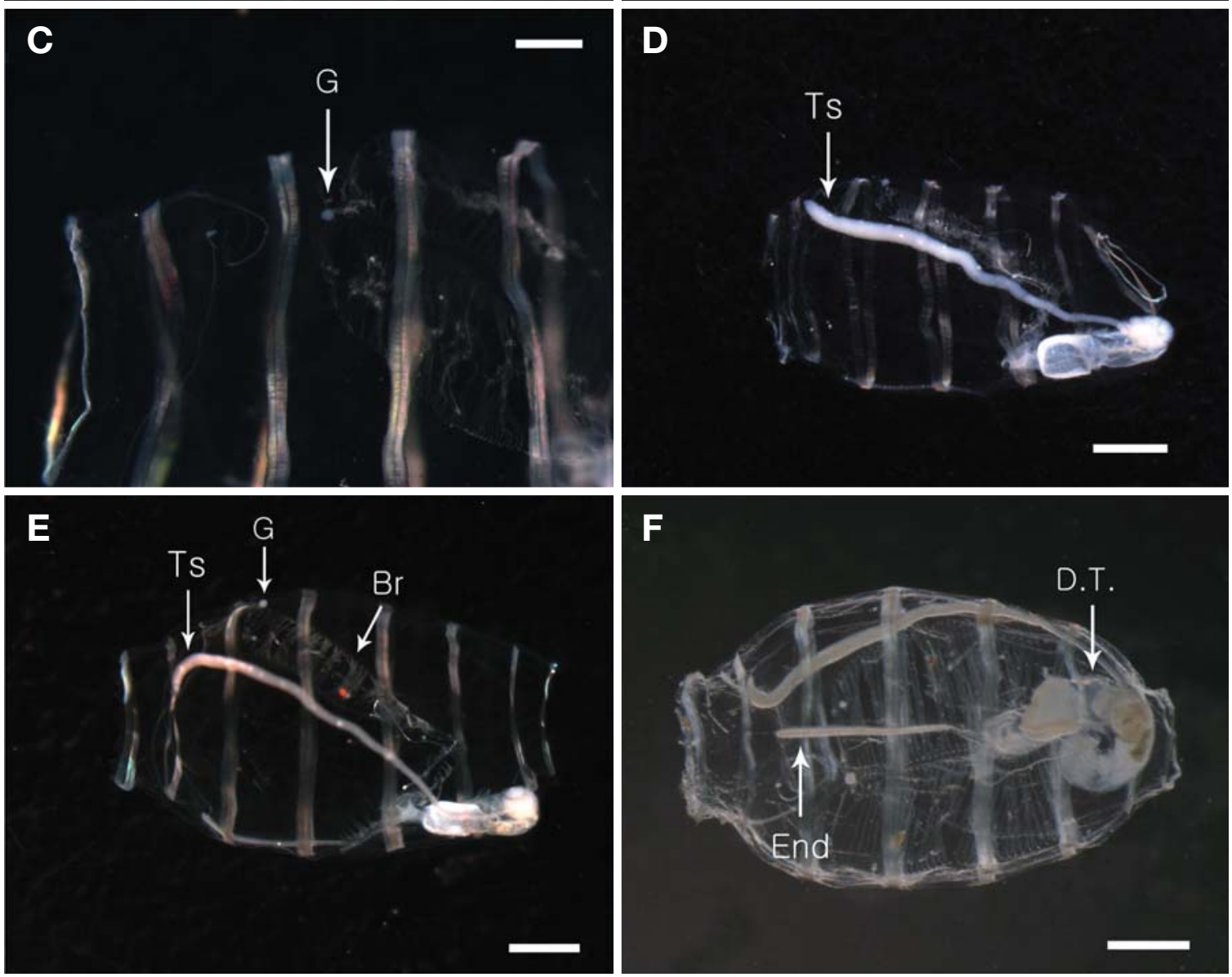

Fig. 2. A-D. Dolioletta gegenbauri, phorozooid stage. $A$, lateral view; $B$, ventral view; $C$, ganglion; $D$, testis of gonozooid stage. $E-$ F. Dolioletta tritonis, gonozooid stage. $\mathrm{E}$, lateral view; $\mathrm{F}$, ventral view. (Br, branchial septum; D.T., intestine; End, endostyle; G, neural ganglion; Ts, testis). Scale bars $=2 \mathrm{~mm}(A-B, D-F), 1 \mathrm{~mm}(C)$.

Doliolum (Dolioletta) gegenbauri: Berrill, 1950, p. 286, fig. 103.

Doliotetta gegenbauri: Garstang, 1933 (cited from Godeaux, 1998a); Madin, 1991, p. 107, fig. T-2.
Material examined. 6 individuals, South Sea, $34^{\circ} 05^{\prime} \mathrm{N} 127^{\circ}$ $96^{\prime} \mathrm{E}, 4$ Oct. 2007 ; 3 individuals, Yellow Sea, $35^{\circ} 58^{\prime} \mathrm{N} 125^{\circ}$ $32^{\prime} \mathrm{E}, 20$ Nov. 2008; 2 individuals, East Sea, $35^{\circ} 31^{\prime} \mathrm{N} 129^{\circ}$ 33'E, 27 Feb. 2009. 
Description. Phorozooid stage: $3-15 \mathrm{~mm}$ long, transparent barrel-shaped body with eight muscle bands (Fig. 2A). Branchial septum from M III dorsally to M V ventrally (Fig. 2A). Endostyle M II 1/2-M IV 1/2 (Fig. 2B). Intestine dextrally coiled (Fig. 2B). Neural ganglion at M III 1/4 (Fig. 2C).

Gonozooid stage: Testis tubular, oblique, start from M II, sometimes beyond M II (Fig. 2D).

Remarks. This species is newly reported from Korea.

Distribution. Atlantic Ocean, tropical and temperate southwestern Indian Ocean, tropical West and southwestern Pacific Ocean, Mediterranean Sea.

Dolioletta tritonis (Herdman, 1888) (Fig. 2E, F)

Doliolum tritonis Herdman, 1888, p. 1 (cited from Berrill, 1950).

Dolioletta tritonis: Garstang, 1933 (cited from Godeaux, 1998a).

Dolioletta gegenbaueri var. tritonis: Chihara and Maurano, 1997, p. 1358.

Material examined. 15 individuals, South Sea, $34^{\circ} 05^{\prime} \mathrm{N} 127^{\circ}$ 96'E, 19 Jun. 2007 and 12 Mar. 2008.

Description. Gonozooid stage: $3-15 \mathrm{~mm}$ long transparent barrel-shaped body with eight muscle bands (Fig. 2E). Branchial septum from M III dorsally to M IV $1 / 2$ ventrally (Fig. 2E). Endostyle M II 1/2 - M IV 1/2 (Fig. 2F). Intestine dextrally coiled (Fig. 2F). Neural ganglion at M III 1/4 (Fig. 2E). Testis tubular, oblique, start from M II, sometimes beyond M II (Fig. 2E).

Remarks. D. tritonis is very similar to D. gegenbauri except branchial septum extending from M III dorsally to M VI 1/2 ventrally.

Distribution. Atlantic Ocean, tropical and temperate Southwestern Indian Ocean, tropical West and Southwestern Pacific Ocean, Mediterranean Sea.

\section{A Key to the species of family Doliolidae from Korea (phorozooid and gonozooid stages)}

1. Intestine arched dextrally …................ 2(Doliolum)

- Intestine loop close coiled …................. 3 (Dolioletta)

2. Branchial septum strongly arched from M II dorsally to
M III ventrally, endostyle short M II-M IV

Doliolum denticulatum

- Branchial septum arched from M II dorsally to just in front of M V ventrally, endostyle short M II-M IV

Doliolum nationalis

3. Branchial septum from M III dorsally to M V ventrally, endostyle M II 1/2-M IV 1/2 ….... Dolioletta gegenbauri

- Branchial septum from M III dorsally to M IV $1 / 2$ ventrally, endostyle M II 1/2-M IV 1/2 ….... Dolioletta tritonis

\section{ACKNOWLEDGEMENTS}

We thank Ecocean Inc. for providing the specimens examined in this work. We appreciate three anonymous reviewers for helpful comments.

\section{REFERENCES}

Berrill, N.J., 1950. The Tunicata, with an Account of the British Species. The Ray Soc. Lond., pp. 1-354.

Chihara, M. and M. Maurano, 1997. An Illustrated Guide to Marine Plankton in Japan. Tokai Univ. Press, Tokyo, pp. 11574.

Godeaux, J., 1998a. The genus Doliolina (Thaliacea: Doliolida). J. Plankton Res., 20(9): 1757-1766.

Godeaux, J., 1998b. The relationships and systematics of the Thaliacea, with keys for identification. In: The biology of pelagic tunicates (Ed., Q. Bone). pp. 273-294. Oxford Univ. Press, Oxford.

Kott, P., 2005. Catalogue of Tunicata in Australian Waters. Commonw. Aust. Canberra, pp. 1-301.

Madin, L.P., 1991. Distribution and taxonomy of zooplankton in the Alboran Sea and adjacent western Mediterranean. A literature survey and field guide. Woods Hole Oceanogr. Inst. MA, pp. 1-147.

The Korean Society of Systematic Zoology, 1997. List of Animals in Korea (Excluding Insects). Academy Publ. Co., Seoul, pp. 1-489. 